\title{
ON THE ASYMPTOTIC EXPANSION OF THE LOGARITHM OF BARNES TRIPLE GAMMA FUNCTION
}

\author{
STAMATIS KOUMANDOS and HENRIK L. PEDERSEN
}

\begin{abstract}
It is shown that the remainders in an asymptotic expansion of the logarithm of Barnes triple gamma function give rise to completely monotonic functions. Furthermore, error bounds are found.
\end{abstract}

\section{Introduction and results}

This paper deals with Barnes' triple gamma function. We recall the definitions of the multiple gamma functions. The multiple zeta function $\zeta_{N}$ with parameters $a_{1}, \ldots, a_{N}$ is defined as

$$
\zeta_{N}(z, w)=\sum_{m_{1}, \ldots, m_{N}=0}^{\infty}\left(w+a_{1} m_{1}+\cdots+a_{N} m_{N}\right)^{-z}
$$

for $\Re z>N$ and $\Re w>0$. Regarded as a function of $z, \zeta_{N}(z, w)$ has a meromorphic extension to the complex plane with simple poles only at $z=$ $1, \ldots, N$. The logarithm of the multiple gamma function is defined as

$$
\log \Gamma_{N}\left(w \mid a_{1}, \ldots, a_{N}\right)=\left.\partial_{z} \zeta_{N}(z, w)\right|_{z=0} .
$$

The multiple zeta and gamma functions have been introduced by E. W. Barnes in a series of papers, published in the beginning of the twentieth century, where several properties of these functions and applications to the theory of elliptic and theta functions have been obtained. Over the years, many researchers have also studied these functions, largely due to their importance in analytic number theory and mathematical physics. The functions are related to Selberg zeta functions and determinants of Laplacians occurring in symmetric space theory. S. N. M. Ruijsenaars gave in [18] complete asymptotic expansions for multiple zeta and gamma functions in terms of an expression involving powers

Received August 13, 2008, in revised form January 21, 2009. 
of $1 / w$ and generalized Bernoulli polynomials. We also refer to [18] and the references given therein for background information on the subject.

In this work, we investigate Ruijsenaars' asymptotic expansion of the logarithm of the triple gamma function with parameters $a_{1}=a_{2}=a_{3}=1$. It is given as follows.

$$
\begin{aligned}
\log \Gamma_{3}(w \mid 1,1,1)= & \frac{B_{3,3}(w)}{6} \log w-\frac{11}{36} B_{3,0} w^{3}-\frac{3}{4} B_{3,1} w^{2}-\frac{1}{2} B_{3,2} w \\
& +\sum_{k=4}^{m} \frac{(-1)^{k}}{k !}(k-4) ! B_{3, k} w^{3-k}+R_{3, m}(w),
\end{aligned}
$$

where the triple Bernoulli polynomials $B_{3, k}(x)$ are defined by

$$
\frac{t^{3} e^{x t}}{\left(e^{t}-1\right)^{3}}=\sum_{k=0}^{\infty} B_{3, k}(x) \frac{t^{k}}{k !}, \quad|t|<2 \pi
$$

and the triple Bernoulli numbers by $B_{3, k}=B_{3, k}(0)$.

The remainder $R_{3, m}$ of order $m$ has the representation

$$
R_{3, m}(w)=\int_{0}^{\infty} \frac{e^{-w t}}{t^{4}}\left(\frac{t^{3}}{\left(1-e^{-t}\right)^{3}}-\sum_{k=0}^{m} \frac{(-1)^{k}}{k !} B_{3, k} t^{k}\right) d t .
$$

Here, $\Re w>0$ and $m \geq 3$. See [18, (3.13) and (3.14)]. Our main results concern the remainders $R_{3, m}(x), x>0$ and they are given in Theorems 1.3, 1.4 and 1.5 below. Before stating them we recall the definition of a completely monotonic function.

Definition 1.1. A function $f:(0, \infty) \rightarrow \mathrm{R}$ is called completely monotonic if $f$ is infinitely often differentiable and

$$
(-1)^{n} f^{(n)}(x) \geq 0
$$

for $x>0$ and for $n \geq 0$.

It is known that if a non constant function $f$ is completely monotonic then strict inequality holds in (3), see [8] and [9]. Such a function is positive, decreasing and convex (a typical example is $f(x)=1 / x^{a}$ for $a>0$ ). A fundamental result of Bernstein relates completely monotonic functions with the Laplace transform (see [20, p. 161]).

THEOREM 1.2 (Bernstein). A function $f$ is completely monotonic if and only if

$$
f(x)=\int_{0}^{\infty} e^{-s x} d \mu(s),
$$


where $\mu$ is a positive measure on $[0, \infty)$ making the integral converge for any $x>0$.

We remark that completely monotonic functions appear in e.g. potential theory in connection with convolution semigroups. See [7].

Our first result deals with complete monotonicity of the even indexed remainders $R_{3,2 n}$. This settles a conjecture stated by the first named author in [13, Conjecture 2].

THEOREM 1.3. For $n \geq 2$ the remainder $(-1)^{n} R_{3,2 n}(x)$ has the representation

$$
(-1)^{n} R_{3,2 n}(x)=\int_{0}^{\infty} e^{-x t} t^{2 n-3}(-1)^{n} v_{n}(t) d t,
$$

where $v_{n}(t)$ is defined in Lemma 2.3 below.

For $n \geq 6$ the remainder $(-1)^{n} R_{3,2 n}(x)$ is a completely monotonic function. For $n \leq 5$ it is not completely monotonic.

It has already been pointed out in $[18, \mathrm{p} .118]$ that the above remainder satisfies

$$
R_{3, m}(x)=O\left(x^{2-m}\right), \quad x \rightarrow \infty .
$$

Here we provide a sharp upper estimate for $(-1)^{n} R_{3,2 n}(x)$.

THEOREM 1.4. For $n \geq 5$ the following error bound holds

$$
\begin{aligned}
(-1)^{n} R_{3,2 n}(x)< & \frac{\left|B_{3,2 n+1}\right|}{(2 n-2)(2 n-1)(2 n)(2 n+1)} \frac{1}{x^{2 n-2}} \\
& +\frac{\left|B_{3,2 n+2}\right|}{(2 n-1)(2 n)(2 n+1)(2 n+2)} \frac{1}{x^{2 n-1}} .
\end{aligned}
$$

This bound is sharp as $x \rightarrow \infty$ in the sense that

$$
\lim _{x \rightarrow \infty}(-1)^{n} R_{3,2 n}(x) x^{2 n-2}=\frac{\left|B_{3,2 n+1}\right|}{(2 n-2)(2 n-1)(2 n)(2 n+1)} .
$$

We also obtain some information about the remainders of odd order.

THEOREM 1.5. For $n \geq 1$ the remainder $(-1)^{n-1} R_{3,2 n+1}(x)$ has the representation

$$
(-1)^{n-1} R_{3,2 n+1}(x)=\int_{0}^{\infty} e^{-x t} t^{2 n-3}(-1)^{n-1} \lambda_{n}(t) d t,
$$

where

$$
\lambda_{n}(t)=v_{n}(t)+\frac{B_{3,2 n+1}}{(2 n+1) !}, \quad n=1,2, \ldots
$$


For $1 \leq n \leq 4$ the remainder $(-1)^{n-1} R_{3,2 n+1}(x)$ is a completely monotonic function. For $n \geq 5$ it is not completely monotonic.

In all cases the following error bound holds

$$
\begin{aligned}
\left|R_{3,2 n+1}(x)\right|< & \frac{\left|B_{3,2 n+2}\right|}{(2 n-1)(2 n)(2 n+1)(2 n+2)} \frac{1}{x^{2 n-1}} \\
& +\frac{\left|B_{3,2 n+3}\right|}{(2 n)(2 n+1)(2 n+2)(2 n+3)} \frac{1}{x^{2 n}} \\
& +\frac{\left|B_{3,2 n+5}\right|}{(2 n+2)(2 n+3)(2 n+4)(2 n+5)} \frac{1}{x^{2 n+1}} .
\end{aligned}
$$

REMARKs 1.6. 1) Theorem 1.3 states in particular that $(-1)^{n} R_{3,2 n}(x)$ is a positive function on $(0, \infty)$ for $n \geq 6$ and thus the left hand side of the inequality in Theorem 1.4 can be replaced by $\left|R_{3,2 n}(x)\right|$, when $n \geq 6$.

2) The asymptotic behaviour as $x$ tends to 0 is found to be

$$
\lim _{x \rightarrow 0_{+}}(-1)^{n} R_{3,2 n}(x) x^{2 n-3}=\frac{(-1)^{n} B_{3,2 n}}{(2 n-3)(2 n-2)(2 n-1) 2 n} .
$$

(For a derivation see Remark 3.6.) The error bound (Theorem 1.4) is thus of a different magnitude in $x$ as $x$ tends to 0 . If one replaces $n$ by $n-1$ in the term

$$
\frac{\left|B_{3,2 n+2}\right|}{(2 n-1)(2 n)(2 n+1)(2 n+2)} \frac{1}{x^{2 n-1}}
$$

in the error bound one obtains the asymptotic behaviour as $x$ tends to 0 .

The proofs of Theorems 1.3-1.5 are based on a suitable representation of the integrand

$$
\frac{t^{3}}{\left(1-e^{-t}\right)^{3}}-\sum_{k=0}^{2 n} \frac{(-1)^{k}}{k !} B_{3, k} t^{k}
$$

in the integral (2). This representation is obtained via Cauchy's residue theorem (see Lemma 2.3). The statement about complete monotonicity of $(-1)^{n} R_{3,2 n}(x)$ for $n \geq 2$ is obtained via Bernstein's theorem by showing that (4) is positive (for $t>0$ ) for $n \geq 6$ and changes sign for $2 \leq n \leq 5$. Here, some results of independent interest on Turán-type inequalities for odd Bernoulli polynomials (see Lemma 2.1) and a new result concerning the monotonicity of the quotient of two series of functions (see Lemma 2.2) are proved.

The proofs of Theorem 1.4 and Theorem 1.5 depend on suitable upper bounds on the integrand above. 
We have earlier investigated the remainders in asymptotic expansions of Euler's gamma function and Barnes double gamma function, see [13], [14], [16] and [17], where analogous results were found. The results obtained in the present paper are technically more difficult.

\section{Preliminary results}

In this section we formulate some preliminary results, some of which are of independent interest, e.g. Turán-type inequalities for odd Bernoulli polynomials and monotonicity properties of a quotient of series of functions. We also state and indicate the proof of the fundamental representation of the integrand (4).

First of all we recall the definition of the ordinary Bernoulli polynomials $B_{k}(x)$ and numbers $B_{k}=B_{k}(0)$ :

$$
\frac{t e^{t x}}{e^{t}-1}=\sum_{k=0}^{\infty} B_{k}(x) \frac{t^{k}}{k !}, \quad|t|<2 \pi .
$$

The triple Bernoulli polynomials are related to the ordinary Bernoulli polynomials by the formula

$$
\begin{aligned}
& B_{3, k}(x) \\
& =\frac{k(k-1)(k-2)}{2}\left\{(x-1)(x-2) \frac{B_{k-2}(x)}{k-2}-(2 x-3) \frac{B_{k-1}(x)}{k-1}+\frac{B_{k}(x)}{k}\right\},
\end{aligned}
$$

see [15, p. 187]. It follows from this that

$$
\frac{B_{3,2 k+1}}{(2 k+1)(2 k-1)}=\frac{3}{2} B_{2 k}
$$

and

$$
\frac{B_{3,2 k+2}}{2 k(2 k+1)(2 k+2)}=\frac{B_{2 k}}{2 k}+\frac{1}{2} \frac{B_{2 k+2}}{(2 k+2)} .
$$

The following lemma furnishes an inequality between (ordinary) Bernoulli polynomials of odd order.

Lemma 2.1. For $n \geq 6$ the following inequality holds

$$
(-1)^{n} B_{2 n-1}(u)>2(-1)^{n-1} B_{2 n-3}(u), \quad 0<u<1 / 2 .
$$

The reverse strict inequality holds when $2 \leq n \leq 5$.

We also need a quite general result about quotients of certain series of functions. 
Lemma 2.2. Suppose that $a_{k}>0, b_{k}>0$ and that $\left\{u_{k}(x)\right\}$ is a sequence of positive $C^{1}$-functions such that the series

$$
\sum_{k=0}^{\infty} a_{k} u_{k}^{(l)}(x) \text { and } \sum_{k=0}^{\infty} b_{k} u_{k}^{(l)}(x), \quad l=0,1
$$

converge absolutely and uniformly over compact subsets of $[0, \infty)$. Define

$$
f(x)=\frac{\sum_{k=0}^{\infty} a_{k} u_{k}(x)}{\sum_{k=0}^{\infty} b_{k} u_{k}(x)} .
$$

- If the logarithmic derivatives $u_{k}^{\prime}(x) / u_{k}(x)$ form an increasing sequence of functions and if $a_{k} / b_{k}$ decreases (resp. increases) then $f(x)$ decreases (resp. increases) for $x \geq 0$.

- If the logarithmic derivatives $u_{k}^{\prime}(x) / u_{k}(x)$ form a decreasing sequence of functions and if $a_{k} / b_{k}$ decreases (resp. increases) then $f(x)$ increases (resp. decreases) for $x \geq 0$.

We now turn to the representation of the integrand in the integral (2) representing the remainder. Up to the factor $e^{w t} / t^{4}$ the integrand is itself a remainder in a certain Taylor expansion. Put

$$
f(t):=\left(\frac{t}{1-e^{-t}}\right)^{3} .
$$

LEMma 2.3. We have, for $t>0$ and $n \geq 2$,

$$
f(t)-\sum_{l=0}^{2 n} \frac{f^{(l)}(0)}{l !} t^{l}=t^{2 n+1} v_{n}(t),
$$

where $v_{n}$ satisfies

$$
\begin{aligned}
&(-1)^{n} v_{n}(t)=\sum_{k=1}^{\infty} \frac{1}{t^{2}+(2 \pi k)^{2}} \frac{1}{(2 \pi k)^{2 n-2}} \cdot\left\{t\left(\frac{(2 n-2)(2 n-1)}{(2 \pi k)^{2}}-2\right)\right. \\
&+\frac{t}{t^{2}+(2 \pi k)^{2}}\left(-3 t-\frac{2\left(t^{2}-(2 \pi k)^{2}\right)}{t^{2}+(2 \pi k)^{2}}+2(2 n-2)\right) \\
&+\frac{2 \pi k}{t^{2}+(2 \pi k)^{2}}\left(6 \pi k+\frac{3(2 n-2)\left(t^{2}+(2 \pi k)^{2}\right)}{2 \pi k}\right. \\
&\left.\left.+\frac{8 \pi k t}{t^{2}+(2 \pi k)^{2}}+\frac{2(2 n-2) t}{2 \pi k}\right)\right\} .
\end{aligned}
$$


Proof of Lemma 2.3. The relation is found using a contour integration argument similar to the arguments in [17]. We consider the auxiliary meromorphic function

$$
g(z):=\frac{f(z)}{(t-z) z^{L}} .
$$

It has a simple pole at $z=t$, a pole of multiplicity $L$ at $z=0$ and triple poles at $z=2 \pi i k, k \neq 0$, assuming that $t$ is different from the poles at 0 and $2 \pi i k$, $k \neq 0$. The corresponding residues at $t$ and 0 are equal to

$$
\begin{aligned}
\operatorname{Res}(g, t) & =-\frac{f(t)}{t^{L}} \\
\operatorname{Res}(g, 0) & =\sum_{l=0}^{L-1} \frac{f^{(l)}(0)}{l !} t^{l-L} .
\end{aligned}
$$

The residue at $z=2 \pi i k$ is computed by the formula

$$
\operatorname{Res}(g, 2 \pi i k)=\frac{1}{2} \lim _{z \rightarrow 2 \pi i k}\left(g(z)(z-2 \pi i k)^{3}\right)^{\prime \prime} .
$$

In this way it follows that

$$
\begin{aligned}
2 \operatorname{Res}(g, 2 \pi i k)=\frac{2}{t-2 \pi i k} \frac{1}{(2 \pi i k)^{L-3}} & \\
& +\frac{3}{t-2 \pi i k} \frac{1}{(2 \pi i k)^{L-3}}\left(\frac{1}{t-2 \pi i k}+\frac{3-L}{2 \pi i k}\right) \\
& +\frac{1}{t-2 \pi i k} \frac{1}{(2 \pi i k)^{L-3}}\left(\frac{2}{(t-2 \pi i k)^{2}}\right. \\
& \left.\quad+\frac{2(3-L)}{(t-2 \pi i k) 2 \pi i k}+\frac{(3-L)(2-L)}{(2 \pi i k)^{2}}\right) .
\end{aligned}
$$

Since $\operatorname{Res}(g,-2 \pi i k)=\overline{\operatorname{Res}(g, 2 \pi i k)}$ we find

$$
\begin{aligned}
\sum_{k \neq 0} \operatorname{Res}(g,-2 \pi i k) \\
=\sum_{k=1}^{\infty} 2 \Re \operatorname{Res}(g, 2 \pi i k) \\
=\sum_{k=1}^{\infty} \Re\left\{\frac { 1 } { t - 2 \pi i k } \frac { 1 } { ( 2 \pi i k ) ^ { L - 3 } } \left(2+\frac{3}{t-2 \pi i k}+\frac{3(3-L)}{2 \pi i k}\right.\right. \\
\left.\left.\quad+\frac{2}{(t-2 \pi i k)^{2}}+\frac{2(3-L)}{(t-2 \pi i k) 2 \pi i k}+\frac{(3-L)(2-L)}{(2 \pi i k)^{2}}\right)\right\} .
\end{aligned}
$$


This sum is evaluated for $L=2 n+1$ by finding the real- and imaginary parts of the factors. The computation is standard, but care has to be taken in order to group the terms suitably.

Then Cauchy's residue theorem is invoked where one integrates along the boundary of the rectangle $\mathscr{E}_{K}=[-K, K] \times[-2 \pi i(K+1 / 2), 2 \pi i(K+1 / 2)]$ (where $K$ is a positive integer) and lets $K$ tend to infinity.

In the investigation of the remainders in an asymptotic expansion of the logarithm of Euler's gamma function the representation similar to (7) is

(8) $\frac{t}{e^{t}-1}=1-\frac{t}{2}+\sum_{k=1}^{n} \frac{B_{2 k}}{(2 k) !} t^{2 k}+(-1)^{n} t^{2 n+2} V_{n}(t), \quad$ for all $\quad t>0$,

where the term $V_{n}(t)$ is given as (see [19, p. 64])

$$
V_{n}(t)=\sum_{k=1}^{\infty} \frac{2}{\left(t^{2}+4 \pi^{2} k^{2}\right)(2 \pi k)^{2 n}}, \quad n \geq 0 .
$$

We notice that

$$
V_{n}(0)=(-1)^{n} \frac{B_{2 n+2}}{(2 n+2) !},
$$

which can be seen directly from (8) or from the well-known formula

$$
\zeta(2 n)=\frac{(-1)^{n-1}(2 \pi)^{2 n}}{2(2 n) !} B_{2 n}, \quad n=1,2, \ldots
$$

As mentioned in the introduction, it is the representation in Lemma 2.3 that makes it possible for us to show that $(-1)^{n} v_{n}(t)>0$ for $n \geq 6$ and hence that $(-1)^{n} R_{3,2 n}(x)$ is completely monotonic for these values of $n$. See Lemma 3.2 and Lemma 3.3.

The remainder $t^{2 n+1} v_{n}(t)$ can also be expressed in terms of the functions $V_{n}(t)$ and $V_{n-1}(t)$, defined in (9). This has already been noticed in [13, Section 3] and the relation is

$$
\begin{aligned}
(-1)^{n} v_{n}(t)=-t & V_{n-1}(t)+\frac{3}{2}(2 n-1) V_{n-1}(t)+\frac{3}{2} t V_{n-1}^{\prime}(t) \\
& +n(2 n+1) t V_{n}(t)+(2 n+1) t^{2} V_{n}^{\prime}(t)+\frac{1}{2} t^{3} V_{n}^{\prime \prime}(t),
\end{aligned}
$$

for $t>0$ and $n \geq 2$. Relation (12) is used in proving the error bound in Theorem 1.4.

We remark that it has not been possible for us to obtain positivity of $(-1)^{n} v_{n}(t)$ from (12). The terms are not grouped in the "right" way. On the other hand, the representation in (7) does not seem well suited for upper bounds. 


\section{Proof of the main results}

Notice that

$$
(-1)^{n} v_{n}(t)=\xi_{n}(t)+\eta_{n}(t)
$$

where

$$
\xi_{n}(t)=t \sum_{k=1}^{\infty} \frac{1}{t^{2}+(2 \pi k)^{2}} \frac{1}{(2 \pi k)^{2 n-2}}\left(\frac{(2 n-2)(2 n-1)}{(2 \pi k)^{2}}-2\right)
$$

and

$$
\begin{aligned}
& \eta_{n}(t) \\
& =\sum_{k=1}^{\infty} \frac{1}{\left(t^{2}+(2 \pi k)^{2}\right)^{2}} \frac{1}{(2 \pi k)^{2 n-2}}\left\{t\left(-3 t-\frac{2\left(t^{2}-(2 \pi k)^{2}\right)}{t^{2}+(2 \pi k)^{2}}+2(2 n-2)\right)\right. \\
& \left.+2 \pi k\left(6 \pi k+\frac{3(2 n-2)\left(t^{2}+(2 \pi k)^{2}\right)}{2 \pi k}+\frac{8 \pi k t}{t^{2}+(2 \pi k)^{2}}+\frac{2(2 n-2) t}{2 \pi k}\right)\right\} .
\end{aligned}
$$

We now state some key lemmas. They are proved in the next section.

LEMMA 3.1. We have

$$
\frac{\zeta(2 n)}{\zeta(2 n-2)}>\frac{(2 \pi)^{2}}{(n-1)(2 n-1)}
$$

for $n \geq 6$ and the reverse strict inequality holds for $2 \leq n \leq 5$.

Lemma 3.2. The expression $\eta_{n}(t)$ is positive for $t>0$ and $n \geq 2$.

Lemma 3.3. The expression $\xi_{n}(t)$ is positive for $t>0$ and $n \geq 6$.

Proof of Theorem 1.3. The positivity of $(-1)^{n} v_{n}(t)$ for $n \geq 6$ follows from Lemma 3.2 and 3.3. Therefore,

$$
(-1)^{n} R_{3,2 n}(x)=\int_{0}^{\infty} e^{-x t} t^{2 n-3}(-1)^{n} v_{n}(t) d t
$$

is completely monotonic for $n \geq 6$ in view of Theorem 1.2.

For $2 \leq n \leq 5$ the function $(-1)^{n} v_{n}(t)$ changes its sign on the positive axis. Indeed,

$$
(-1)^{n} v_{n}(0)=3(2 n-1) \frac{\zeta(2 n)}{(2 \pi)^{2 n}}>0
$$

and

$$
\lim _{t \rightarrow \infty} t(-1)^{n} v_{n}(t)=(2 n-2)(2 n-1) \frac{\zeta(2 n)}{(2 \pi)^{2 n}}-2 \frac{\zeta(2 n-2)}{(2 \pi)^{2 n-2}}<0
$$


by Lemma 3.1. Therefore $(-1)^{n} R_{3,2 n}(x)$ is not completely monotonic for these values of $n$, by Theorem 1.2.

Next we turn to the error bound. Here the following lemma about the functions $V_{n}$ is essential.

LemMa 3.4. Let

$$
\Psi_{n}(t):=-\left[(4 n+2) V_{n}^{\prime}(t)+t V_{n}^{\prime \prime}(t)\right]
$$

and

$$
\Phi_{n}(t):=n(2 n+1) V_{n}(t)-V_{n-1}(t) .
$$

Then the following inequalities hold for $t>0$ :

$$
0<\Psi_{n}(t)<2 t(4 n+3)(-1)^{n-1} \frac{B_{2 n+4}}{(2 n+4) !}, \quad \text { for all } n \geq 0
$$

and

$$
0<\Phi_{n}(t)<\Phi_{n}(0), \quad \text { for all } n \geq 5
$$

while

$$
\Phi_{n}(0)<\Phi_{n}(t)<0, \quad \text { for all } 1 \leq n \leq 4
$$

Lemma 3.5. The following inequalities hold

$$
\begin{aligned}
(-1)^{n-1} B_{3,2 n+1} & >0, & & \geq \geq 0, \\
(-1)^{n} B_{3,2 n+2} & >0, & & n \geq 5, \\
(-1)^{n} B_{3,2 n+2} & <0, & & 1 \leq n \leq 4 .
\end{aligned}
$$

Proof OF Theorem 1.4. Using (13) and (14) in (12) we find that

$$
\begin{aligned}
& (-1)^{n} t^{2 n-3} v_{n}(t) \\
& \quad=-\frac{1}{2} \Psi_{n}(t) t^{2 n-1}+\frac{3}{2} V_{n-1}^{\prime}(t) t^{2 n-2}+\frac{3}{2}(2 n-1) V_{n-1}(t) t^{2 n-3}+\Phi_{n}(t) t^{2 n-2} \\
& \quad<\frac{3}{2}(2 n-1) V_{n-1}(0) t^{2 n-3}+\Phi_{n}(0) t^{2 n-2} \\
& \quad=\frac{3}{2}(2 n-1) V_{n-1}(0) t^{2 n-3}+\left[n(2 n+1) V_{n}(0)-V_{n-1}(0)\right] t^{2 n-2} .
\end{aligned}
$$


We deduce from this and relation (10) that, for $n \geq 5$,

$$
\begin{aligned}
(-1)^{n} R_{3,2 n}(x)= & \int_{0}^{\infty} e^{-x t} t^{2 n-3}(-1)^{n} v_{n}(t) d t \\
< & \frac{3}{2} \frac{(-1)^{n-1} B_{2 n}}{(2 n-2)(2 n)} \frac{1}{x^{2 n-2}} \\
& +(-1)^{n}\left\{\frac{1}{2} \frac{B_{2 n+2}}{(2 n-1)(2 n+2)}+\frac{B_{2 n}}{(2 n-1)(2 n)}\right\} \frac{1}{x^{2 n-1}} \\
= & \frac{\left|B_{3,2 n+1}\right|}{(2 n-2)(2 n-1)(2 n)(2 n+1)} \frac{1}{x^{2 n-2}} \\
& +\frac{\left|B_{3,2 n+2}\right|}{(2 n-1)(2 n)(2 n+1)(2 n+2)} \frac{1}{x^{2 n-1}} .
\end{aligned}
$$

We have used (5), (6) together with Lemma 3.5 in order to obtain the last equality. The proof of the error bound is complete.

The asymptotic behaviour as $x$ tends to infinity follows directly from the expansion (1). Indeed,

$$
R_{3, m}(x)=\frac{(-1)^{m+1}}{(m+1) !}(m-3) ! B_{3, m+1} x^{2-m}+R_{3, m+1}(x),
$$

where (as noticed before) $R_{3, k}(x)=O\left(x^{2-k}\right)$ as $x \rightarrow \infty$. Therefore

$$
\lim _{x \rightarrow \infty} R_{3, m}(x) x^{m-2}=\frac{(-1)^{m+1}}{(m+1) !}(m-3) ! B_{3, m+1} .
$$

This proves the result.

REMARK 3.6. (Proof of the asymptotic behaviour of $R_{3,2 n}(x)$ as $x$ tends to 0.) We notice

$$
\left|(-1)^{n} v_{n}(t)\right| \leq \sum_{k=1}^{\infty} \frac{\operatorname{Const}(n)}{(2 \pi k)^{2 n-2}}<\infty,
$$

and hence for any $s>0$ we have using Lebesgue's theorem on dominated convergence

$$
\begin{aligned}
\lim _{x \rightarrow 0_{+}}(-1)^{n} v_{n}(s / x) s / x & =\lim _{t \rightarrow \infty}(-1)^{n} v_{n}(t) t \\
& =\sum_{k=1}^{\infty} \frac{1}{(2 \pi k)^{2 n-2}}\left(\frac{(2 n-2)(2 n-1)}{(2 \pi k)^{2}}-2\right) \\
& =(2 n-2)(2 n-1) \frac{\zeta(2 n)}{(2 \pi)^{2 n}}-2 \frac{\zeta(2 n-2)}{(2 \pi)^{2 n-2}},
\end{aligned}
$$


and this gives

$$
\begin{aligned}
\lim _{x \rightarrow 0_{+}} & (-1)^{n} R_{3,2 n}(x) x^{2 n-3} \\
& =\lim _{x \rightarrow 0_{+}} \int_{0}^{\infty} e^{-s} s^{2 n-4}(-1)^{n} v_{n}(s / x) s / x d s \\
& =(2 n-4) !\left((2 n-2)(2 n-1) \frac{\zeta(2 n)}{(2 \pi)^{2 n}}-2 \frac{\zeta(2 n-2)}{(2 \pi)^{2 n-2}}\right),
\end{aligned}
$$

which by (6) proves the asserted relation.

Proof of TheOREM 1.5. We first give an expression of $(-1)^{n-1} \lambda_{n}(t)$ in terms of the functions $V_{n}(t)$ and $V_{n-1}(t)$ which is analogous of (12). Using (5) and (10) we obtain

$$
(-1)^{n-1} \frac{B_{3,2 n+1}}{(2 n+1) !}=\frac{3}{2}(2 n-1) V_{n-1}(0) .
$$

Then by the recurrence formula

$$
t^{2} V_{n}(t)=V_{n-1}(0)-V_{n-1}(t),
$$

see [13] or [14, Proposition 2.5(i)], we deduce that

$$
(-1)^{n-1} \frac{B_{3,2 n+1}}{(2 n+1) !}=\frac{3}{2}(2 n-1) V_{n-1}(t)+\frac{3}{2}(2 n-1) t^{2} V_{n}(t) .
$$

Combining this with (12) we find that

$$
\begin{aligned}
(-1)^{n-1} \lambda_{n}(t)= & t V_{n-1}(t)+\frac{3}{2}(2 n-1) t^{2} V_{n}(t)-\frac{3}{2} t V_{n-1}^{\prime}(t) \\
& -n(2 n+1) t V_{n}(t)-(2 n+1) t^{2} V_{n}^{\prime}(t)-\frac{1}{2} t^{3} V_{n}^{\prime \prime}(t) . \\
=t & {\left[-\Phi_{n}(t)+\frac{t}{2} \Psi_{n}(t)+\frac{3}{2}(2 n-1) t V_{n}(t)-\frac{3}{2} V_{n-1}^{\prime}(t)\right], }
\end{aligned}
$$

where $\Phi_{n}(t)$ and $\Psi_{n}(t)$ are the functions defined in Lemma 3.4.

It follows from (17) that

$$
\lim _{t \rightarrow 0_{+}} \frac{(-1)^{n-1} \lambda_{n}(t)}{t}=-\Phi_{n}(0)=\frac{(-1)^{n-1} B_{3,2 n+2}}{(2 n+2) !}<0
$$

for all $n \geq 5$ according to the second inequality of Lemma 3.5. On the other hand, for $n \geq 5$, we also have

$$
\lim _{t \rightarrow \infty}(-1)^{n-1} \lambda_{n}(t)=\frac{(-1)^{n-1} B_{3,2 n+1}}{(2 n+1) !}>0,
$$


by the first inequality of Lemma 3.5. Taking into account the last two results, we infer that the function $(-1)^{n-1} \lambda_{n}(t)$ changes sign on the positive axis when $n \geq 5$, and therefore the remainder $(-1)^{n-1} R_{3,2 n+1}(x)$ is not completely monotonic for these values of $n$. It is, however, completely monotonic when $1 \leq n \leq 4$. Indeed, this follows immediately using the last expression in (17) and invoking (13) and (15) of Lemma 3.4.

The proof of the error bound uses a similar method as Theorem 1.4 taking into consideration (17). Indeed, by (16) we obtain

$$
\begin{aligned}
\frac{3}{2}(2 n & -1) t V_{n}(t)-\frac{3}{2} V_{n-1}^{\prime}(t) \\
& =\frac{3}{2}(2 n+1) t V_{n}(t)+\frac{3}{2} t^{2} V_{n}^{\prime}(t)<\frac{3}{2}(2 n+1) t V_{n}(0) \\
& =\frac{3}{2}(2 n+1) t(-1)^{n} \frac{B_{2 n+2}}{(2 n+2) !} .
\end{aligned}
$$

From the second inequality in (13) we have

$$
\frac{t}{2} \Psi_{n}(t)<t^{2}(4 n+3)(-1)^{n-1} \frac{B_{2 n+4}}{(2 n+4) !} .
$$

In view of (14) and (15) we have, for all $n \geq 1$,

$$
\left|\Phi_{n}(t)\right| \leq\left|\Phi_{n}(0)\right|=\frac{\left|B_{3,2 n+2}\right|}{(2 n+2) !} .
$$

Using (19), (20), (21) in (17) we conclude that

$$
t^{2 n-3}\left|\lambda_{n}(t)\right|<\frac{\left|B_{3,2 n+2}\right|}{(2 n+2) !} t^{2 n-2}+\frac{\left|B_{3,2 n+3}\right|}{(2 n+3) !} t^{2 n-1}+\frac{2}{3} \frac{4 n+3}{2 n+3} \frac{\left|B_{3,2 n+5}\right|}{(2 n+5) !} t^{2 n},
$$

whence the desired error estimate follows.

\section{Proof of various lemmas}

Proof of Lemma 2.1. We recall that for all $n \geq 1$ we have $(-1)^{n} B_{2 n-1}(u)>$ $0,0<u<1 / 2$. Therefore, for $0<u<1 / 2$, the assertion of the lemma is equivalent to

$$
-\frac{B_{2 n-1}(u)}{B_{2 n-3}(u)}>2, \quad 0<u<1 / 2, \quad n \geq 6 .
$$

It is shown in [11, Prop. 3] that Turán's inequality holds for the odd Bernoulli polynomials, viz.

$$
B_{2 n+1}(u) B_{2 n-3}(u)-B_{2 n-1}(u)^{2}>0, \quad n \geq 2,
$$


for $0<u<1 / 2$ (and by symmetry also for $1 / 2<u<1$ ). This means that the sequence

$$
A_{n}(u):=-\frac{B_{2 n-1}(u)}{B_{2 n-3}(u)}
$$

is strictly increasing for $0<u<1 / 2$ for $n \geq 2$. Hence for $n \geq 6$ we have

$$
A_{n}(u)=-\frac{B_{2 n-1}(u)}{B_{2 n-3}(u)}>A_{6}(u)=-\frac{B_{11}(u)}{B_{9}(u)}>\frac{25}{9}>2,
$$

whence the desired inequality (22) follows. In the cases where $2 \leq n \leq 5$ the inequalities follow by an elementary computation.

Proof of Lemma 3.1. Using (11) and (6) we obtain the identity

$$
\frac{(2 n-2)(2 n-1) \zeta(2 n)}{(2 \pi)^{2 n}}-\frac{2 \zeta(2 n-2)}{(2 \pi)^{2 n-2}}=(-1)^{n-1} \frac{B_{3,2 n}}{(2 n) !} .
$$

It is now clear that the desired inequality is equivalent to the second inequality of Lemma 3.5.

For $2 \leq n \leq 5$ one can easily check that the strict opposite inequality holds, but this is also equivalent to the third inequality of Lemma 3.5.

Proof of Lemma 3.2. Since

$$
2 \pi k \frac{3(2 n-2)\left(t^{2}+(2 \pi k)^{2}\right)}{2 \pi k} \geq 3(2 n-2) t^{2}
$$

and

$$
-\frac{2\left(t^{2}-(2 \pi k)^{2}\right)}{t^{2}+(2 \pi k)^{2}} \geq-2
$$

it is found that

$$
\begin{aligned}
\eta_{n}(t) \geq & \sum_{k=1}^{\infty} \frac{1}{\left(t^{2}+(2 \pi k)^{2}\right)^{2}} \frac{1}{(2 \pi k)^{2 n-2}}\{t(-3 t-2+2(2 n-2)) \\
& \left.+\left(3(2 \pi k)^{2}+3(2 n-2) t^{2}+\frac{4(2 \pi k)^{2} t}{t^{2}+(2 \pi k)^{2}}+2(2 n-2) t\right)\right\} \\
= & \sum_{k=1}^{\infty} \frac{1}{\left(t^{2}+(2 \pi k)^{2}\right)^{2}} \frac{1}{(2 \pi k)^{2 n-2}}\left\{2(2 n-3) t+3(2 \pi k)^{2}\right. \\
& \left.+3(2 n-3) t^{2}+\frac{4(2 \pi k)^{2} t}{t^{2}+(2 \pi k)^{2}}+2(2 n-2) t\right\} .
\end{aligned}
$$

Therefore, $\eta_{n}(t)>0$ for $t>0$ and $n \geq 2$. 
We present two different proofs of Lemma 3.3. The first proof uses Lemma 2.1 and a representation of $\xi_{n}(t)$ in terms of odd Bernoulli polynomials. The second proof relies on Lemma 2.2, which is proved in the next section.

Proof I of Lemma 3.3. It is readily seen that the inequality $\xi_{n}(t)>0$ is equivalent to

$$
(2 n-1)(2 n-2) V_{n}(t)-2 V_{n-1}(t)>0, \quad t>0,
$$

where $V_{n}(t)$ is defined in (9).

There is another representation (cf. [12]) of $V_{n}(t)$ in terms odd Bernoulli polynomials,

$$
V_{n}(t)=\frac{1}{(2 n+1) !} \frac{1}{e^{t}-1} \int_{0}^{1} e^{t u}(-1)^{n} B_{2 n+1}(u) d u .
$$

Next we use (25) to show that inequality (24) holds for $n \geq 6$. For $0<u<1$, we define the polynomials

$$
P_{n}(u)=(-1)^{n}\left[(2 n-1)(2 n-2) B_{2 n+1}(u)+2 n(2 n+1) 2 B_{2 n-1}(u)\right] .
$$

Using (25) we see that (24) is equivalent to

$$
\int_{0}^{1} e^{t u} P_{n}(u) d u>0, \quad t>0 .
$$

It is easy to see that the polynomials $P_{n}(u)$ satisfy the following properties:

$$
P_{n}(1-u)=-P_{n}(u), \quad P_{n}(0)=P_{n}(1)=P_{n}(1 / 2)=0, \quad n \geq 2 .
$$

Since $e^{t u}$ is an increasing function of $u$ for all $t>0$, in order to establish (26) we need only show that

$$
P_{n}(u)<0, \quad 0<u<1 / 2, \quad n \geq 6 .
$$

From Lemma 2.1 and the well-known relation $B_{k}^{\prime}(u)=k B_{k-1}(u)$ it follows that $P_{n}^{\prime \prime}(u)>0$ for $0<u<1 / 2$. The polynomials $P_{n}(u)$ are thus convex on $(0,1 / 2)$ for all $n \geq 6$ and since $P_{n}(0)=P_{n}(1 / 2)=0$ inequality (27) follows at once.

Remark 4.1. For $n \leq 5$ it follows that $\xi_{n}(t)<0$ for all $t>0$. Indeed, in the case $n=1$ of (24) reduces to $V_{0}(t)>0$, and a direct calculation reveals that $P_{n}(u)>0$ for $0<u<1 / 2$ and $1 \leq n \leq 5$, therefore we have the reverse inequality in (26). 
Proof II of Lemma 3.3. Clearly, $\xi_{n}(t)>0$ for $t>0$ is equivalent to

$$
\frac{\sum_{k=1}^{\infty} \frac{1}{t^{2}+(2 \pi k)^{2}} \frac{1}{(2 \pi k)^{2 n}}}{\sum_{k=1}^{\infty} \frac{1}{t^{2}+(2 \pi k)^{2}} \frac{1}{(2 \pi k)^{2 n-2}}}>\frac{1}{(n-1)(2 n-1)}
$$

for $t>0$. This, in turn, is the same as

$$
\mu_{n}(s) \equiv \frac{\sum_{k=1}^{\infty} \frac{1}{s^{2}+k^{2}} \frac{1}{k^{2 n}}}{\sum_{k=1}^{\infty} \frac{1}{s^{2}+k^{2}} \frac{1}{k^{2 n-2}}}>\frac{(2 \pi)^{2}}{(n-1)(2 n-1)}
$$

for $s>0$.

From Lemma 2.2 it is obtained that $\mu_{n}(s)$ is decreasing on the positive line and therefore

$$
\mu_{n}(s)>\frac{(2 \pi)^{2}}{(n-1)(2 n-1)}
$$

holds for all $s>0$ provided that

$$
\lim _{s \rightarrow \infty} \mu_{n}(s) \geq \frac{(2 \pi)^{2}}{(n-1)(2 n-1)} .
$$

It is readily computed that

$$
\lim _{s \rightarrow \infty} \mu_{n}(s)=\frac{\zeta(2 n)}{\zeta(2 n-2)}
$$

and hence, by Lemma 3.1, $\xi_{n}(t)>0$ for $t>0$ and $n \geq 6$.

Proof of Lemma 3.4. For the proof of (13) we use the representation (9) and, as in [14, Lemma 2.3], we find that for $n \geq 1$ we have

$$
\Psi_{n}(t)=\sum_{k=1}^{\infty} \frac{4 t\left[(4 n-1) t^{2}+(4 n+3)\left(4 k^{2} \pi^{2}\right)\right]}{\left(t^{2}+4 \pi^{2} k^{2}\right)^{3}(2 \pi k)^{2 n}}>0 .
$$

In the case where $n=0$ we have $V_{0}(t)=\frac{1}{t^{2}}\left(\frac{1}{2} t \operatorname{coth} \frac{t}{2}-1\right)$, hence $\Psi_{0}(t)=$ $\frac{1}{4 t^{3}}\left(8+t^{3} \operatorname{coth} \frac{t}{2}-t^{3} \operatorname{coth}^{3} \frac{t}{2}\right)>0$ for $t>0$, by an elementary calculation.

On the other hand, it is easy to see that for $n \geq 1$, we have

$$
\begin{aligned}
\sum_{k=1}^{\infty} \frac{(4 n-1) t^{2}+(4 n+3)\left(4 k^{2} \pi^{2}\right)}{\left(t^{2}+4 \pi^{2} k^{2}\right)^{3}(2 \pi k)^{2 n}} & <(4 n+3) \frac{\zeta(2 n+4)}{(2 \pi)^{2 n+4}} \\
& =(4 n+3)(-1)^{n-1} \frac{B_{2 n+4}}{2(2 n+4) !}
\end{aligned}
$$


For $n=0$ we have $\Psi_{0}(t)<t / 120$, by an elementary computation and this completes the proof of the inequalities in (13).

For the proof of inequalities (14) and (15) we first observe that for all $n \geq 1$ we have $\lim _{t \rightarrow \infty} \Phi_{n}(t)=0$. It is therefore sufficient to show that the function $\Phi_{n}(t)$ is strictly decreasing on $[0, \infty)$ for $n \geq 5$, whereas, it is strictly increasing on $[0, \infty)$ for $1 \leq n \leq 4$. To this end, we use a representation for $V_{n}^{\prime}(t)$ (obtained in [13, (3.1)] using (25)), namely

$$
V_{n}^{\prime}(t)=-\frac{1}{(2 n+1) !} \varphi(t) \int_{0}^{1 / 2} K(t, u)(-1)^{n+1} B_{2 n+1}(u) d u,
$$

where

$$
\varphi(t)=\frac{2 t e^{t}}{\left(e^{t}-1\right)^{2}}
$$

and

$$
K(t, u)=u(1-u)\left[\frac{\sinh t(1-u)}{t(1-u)}-\frac{\sinh t u}{t u}\right] .
$$

Note that for $0<u<1 / 2$, we have $K(t, u)>0$ and $(-1)^{n+1} B_{2 n+1}(u)>0$, for all $n$. Using (28) we see that inequality $\Phi_{n}^{\prime}(t)<0$ is equivalent to

$$
\int_{0}^{1 / 2} K(t, u)\left[(-1)^{n+1} B_{2 n+1}(u)-2(-1)^{n} B_{2 n-1}(u)\right] d u>0,
$$

which is true for $n \geq 5$ because of Lemma 2.1. By the same Lemma we also deduce that $\Phi_{n}^{\prime}(t)>0$, for $1 \leq n \leq 4$.

The proof of Lemma 3.4 is complete.

Proof of Lemma 3.5. The first inequality follows directly from (5). Note that $-B_{3,1}=3 / 2$. For the second and third inequality we recall the second equality in (18) (or use (10) and (6)),

$$
\Phi_{n}(0)=\frac{(-1)^{n} B_{3,2 n+2}}{(2 n+2) !},
$$

where $\Phi_{n}(t)$ is the function defined in Lemma 3.4. Since $\Phi_{n}(0)>0$ for $n \geq 5$ and $\Phi_{n}(0)<0$ for $1 \leq n \leq 4$, the proof of the lemma is complete. 


\section{Proof of Lemma 2.2}

The second proof of Lemma 3.3 requires the quotient

$$
\frac{\sum_{k=1}^{\infty} \frac{1}{t^{2}+k^{2}} \frac{1}{k^{2 n}}}{\sum_{k=1}^{\infty} \frac{1}{t^{2}+k^{2}} \frac{1}{k^{2 n-2}}}
$$

to be decreasing on the positive line. It turns out that this is a special case of Lemma 2.2. We have not been able to find references to a result of the kind and generality in the literature. For the case of power series the result has been known for a long time and has been used to obtain various inequalities for special functions. See for instance, [1], [2], [3], [4], [5], [6] and [10]. A particularly simple proof of this case can be found in [3]. Compare also [10, Th. 4.4] for an analogous result in the case of ratio of polynomials of the same degree.

Next we give a proof of Lemma 2.2.

Proof. We shall only show that $f$ decreases if the sequence of logarithmic derivatives $u_{k}^{\prime} / u_{k}$ increases and $a_{k} / b_{k}$ decreases. By differentiation it follows that $f$ decreases if

$$
\sum_{k=0}^{\infty} a_{k} u_{k}^{\prime}(x) \sum_{k=0}^{\infty} b_{k} u_{k}(x) \leq \sum_{k=0}^{\infty} a_{k} u_{k}(x) \sum_{k=0}^{\infty} b_{k} u_{k}^{\prime}(x)
$$

This inequality is equivalent to

$$
\sum_{n=0}^{\infty} \sum_{k=0}^{n} a_{k} b_{n-k}\left(u_{k}^{\prime}(x) u_{n-k}(x)-u_{n-k}^{\prime}(x) u_{k}(x)\right) \leq 0 .
$$

The expression $d_{n, k}(x)=u_{k}^{\prime}(x) u_{n-k}(x)-u_{n-k}^{\prime}(x) u_{k}(x)$ for $0 \leq k \leq n$ satisfies $d_{n, k}(x)=-d_{n, n-k}(x)$ and since the sequence of functions $u_{k}^{\prime}(x) / u_{k}(x)$ is increasing it follows that $d_{n, k}(x) \leq 0$ for $k \leq n / 2$. Furthermore, since the sequence $a_{k} / b_{k}$ decreases we also have $a_{k} b_{n-k}-a_{n-k} b_{k} \geq 0$ for $k \leq n / 2$.

In order to verify the assertion in the lemma we first consider even $n$, that is $n=2 m$ and find, using the symmetry relation $d_{n, k}(x)=-d_{n, n-k}(x)$,

$$
\begin{aligned}
\sum_{k=0}^{2 m} a_{k} b_{2 m-k} d_{2 m, k}(x) & =\sum_{k=0}^{m-1} a_{k} b_{2 m-k} d_{2 m, k}(x)+\sum_{k=m+1}^{2 m} a_{k} b_{2 m-k} d_{2 m, k}(x) \\
& =\sum_{k=0}^{m-1}\left(a_{k} b_{2 m-k}-a_{2 m-k} b_{k}\right) d_{2 m, k}(x) \leq 0 .
\end{aligned}
$$


If $n$ is odd, hence $n=2 m-1$ it follows similarly that

$$
\begin{aligned}
& \sum_{k=0}^{2 m-1} a_{k} b_{2 m-1-k} d_{2 m-1, k}(x) \\
& \quad=\sum_{k=0}^{m-1} a_{k} b_{2 m-1-k} d_{2 m-1, k}(x)+\sum_{k=m}^{2 m-1} a_{k} b_{2 m-1-k} d_{2 m-1, k}(x) \\
& \quad=\sum_{k=0}^{m-1}\left(a_{k} b_{2 m-1-k}-a_{2 m-1-k} b_{k}\right) d_{2 m-1, k}(x) \leq 0 .
\end{aligned}
$$

Therefore $f$ decreases. In the case where the logarithmic derivatives of $u_{k}(x)$ form a strictly increasing sequence and where $a_{k} / b_{k}$ is also strictly decreasing it follows that $f$ is also strictly decreasing.

REMARK 5.1. The functions $u_{k}(x)$ are in the above application of the lemma given by

$$
u_{k}(x)=\frac{1}{x^{2}+(k+1)^{2}}
$$

and it is easy to see that the logarithmic derivatives form an increasing sequence:

$$
\left(\log u_{k}(x)\right)^{\prime}=-\frac{2 x}{x^{2}+(k+1)^{2}} .
$$

Finally, $a_{k}=1 /(k+1)^{2 n}$ and $b_{k}=1 /(k+1)^{2 n-2}$ so $a_{k} / b_{k}=1 /(k+1)^{2}$ decreases.

ACKNOWLEDGement. We would like to thank professor Matti Vuorinen for calling to our attention the reference [3] and an anonymous referee for pointing out the relevant papers [4], [5] and [6]. We are also indebted to referees for offering valuable suggestions for the improvement of the presentation of the paper.

\section{REFERENCES}

1. Anderson, G. D., Vamanamurthy, M. K., and Vuorinen, M., Topics in special functions II, Conform. Geom. Dyn. 11 (2007), 250-270.

2. Anderson, G. D., Vamanamurthy, M. K., and Vuorinen, M., Generalized convexity and inequalities, J. Math. Anal. Appl. 335 (2007), 1294-1308.

3. Baricz, Á., Generalized Bessel functions of the first kind, PhD thesis, Babes-Bolyai University, Cluj-Napoca 2008.

4. Baricz, Á., Functional inequalities involving special functions, J. Math. Anal. Appl. 319 (2006), 450-459. 
5. Baricz, Á., Functional inequalities involving special functions II, J. Math. Anal. Appl. 327 (2007), 1202-1213.

6. Baricz, Á., Functional inequalities for Galué's generalized modified Bessel functions, J. Math. Inequal. 1 (2007), 183-193.

7. Berg, C., and Forst, G., Potential Theory on Locally Compact Abelian Groups, Ergebnisse der Math. und ihrer Grenzgebiete 87, Springer, Berlin 1975.

8. Dubourdieu, M. J., Sur un théorème de M. S. Bernstein relatif à la transformation de LaplaceStieltjes, Comp. Math. 7 (1939), 96-111.

9. Haeringen, H. van, Completely monotonic and related functions, J. Math. Anal. Appl. 204 (1996), 389-408.

10. Heikkala, V., Vamanamurthy, M. K., and Vuorinen, M., Generalized elliptic integrals, Comput. Methods Funct. Theory 9 (2009), 75-109.

11. Ikeda, Masatoshi, Some inequalities for Bernoulli's polynomials and related functions, Monatsh. Math. 68 (1964), 224-234.

12. Koumandos, S., Remarks on some completely monotonic functions, J. Math. Anal. Appl. 324 (2006) 1458-1461.

13. Koumandos, S., On Ruijsenaars' asymptotic expansion of the logarithm of the double gamma function, J. Math. Anal. Appl. 341 (2008), 1125-1132.

14. Koumandos, S., and Pedersen, H. L., Completely monotonic functions of positive order and asymptotic expansions of the logarithm of Barnes double gamma function and Euler's gamma function, J. Math. Anal. Appl. 355 (2009), 33-40.

15. Nörlund, N. E., Mémoire sur les polynomes de Bernoulli, Acta Math. 43 (1922), 121-196.

16. Pedersen, H. L., On the remainder in an asymptotic expansion of the double gamma function, Mediterr. J. Math. 2 (2005), 171-178.

17. Pedersen, H. L., The remainder in Ruijsenaars'asymptotic expansion of Barnes double gamma function, Mediterr. J. Math. 4 (2007), 419-433.

18. Ruijsenaars, S. N. M., On Barnes'multiple zeta and gamma functions, Adv. Math. 156 (2000), 107-132.

19. Temme, N. M., Special Functions: An Introduction to the Classical Functions of Mathematical Physics, Wiley, New York 1996.

20. Widder, D. V., The Laplace Transform, Princeton Math. Series 6, Princeton Univ. Press, Princeton, 1941.

DEPARTMENT OF MATHEMATICS AND STATISTICS THE UNIVERSITY OF CYPRUS

P. O. BOX 20537

1678 NICOSIA

CYPRUS

E-mail: skoumand@ucy.ac.cy
DEPARTMENT OF BASIC SCIENCES AND ENVIRONMENT MATHEMATICS AND COMPUTER SCIENCE

FACULTY OF LIFE SCIENCES

UNIVERSITY OF COPENHAGEN

40, THORVALDSENSVEJ

DK-1871 FREDERIKSBERG C

DENMARK

E-mail: henrikp@dina.kvl.dk 